\title{
Plasticity of the enteric nervous system in patients with intestinal neuronal dysplasia associated with Hirschsprung's disease: a report of three patients
}

Accepted: 22 November 2002/Published online: 19 December 2003 (C) Springer-Verlag 2003

\begin{abstract}
Intestinal neuronal dysplasia is a controversial form of dysganglionosis that has been described both as an isolated disorder and in association with Hirschsprung's disease. It has been blamed for the bad outcome of bowel function in patients operated on for Hirschsprung's disease. According to various authors, intestinal neuronal dysplasia could be a primary disorder or secondary to other diseases of the bowel. The aim of this study was to assess the plasticity of the enteric nervous system in patients with Hirschsprung's disease-associated intestinal neuronal dysplasia and its ability to evolve spontaneously to normal innervation patterns. Since we prospectively introduced the assessment of the enteric nervous system of the ganglionated bowel in patients operated on for Hirschsprung's disease, 31 patients have been operated on for Hirschsprung's disease in our institution between 1995 and 2002. Among these patients, nine suffered postoperatively from severe constipation and five from bouts of entocolitis. IND was found in eight of them. We studied the evolution of the innervation in three of these patients by repeated serial full-thickness biopsies of the colon. All three patients underwent a colostomy before or after the pull-through procedure. Histopathological assessment of the enteric nervous system was made with conventional acetylcholinesterase histochemistry, rapid acetylcholinesterase histochemistry and immunohistochemistry for the Protein Gene Product 9.5 and the antigen CD56. This evolution was compared with the clinical outcome of bowel function. In our three patients with Hirschsprung's disease-associated intestinal neuronal dysplasia,
\end{abstract}

B. J. Meyrat $(\bowtie)$

Service de Chirurgie Pédiatrique, Centre Hospitalier

Universitaire Vaudois (CHUV),

1011 Lausanne, Switzerland

E-mail: blaise-julien.meyrat@chuv.hospvd.ch

Fax: + 41-21-3143076

R. N. Laurini

Institut Universitaire de Pathologie,

Centre Hospitalier Universitaire

Vaudois (CHUV), Lausanne, Switzerland this form of dysganglionosis evolved to normal innervation patterns within a period ranging from 9 to 18 months. This evolution was accompanied by an improvement of bowel function in all. We conclude that Hirschsprung's disease-associated intestinal neuronal dysplasia can evolve to a normal innervation, at least under certain circumstances such as a colostomy. Specific histopathological techniques are required to assess accurately the enteric nervous system.

Keywords Intestinal neuronal dysplasia ·

Hirschsprung's disease $\cdot$ Enteric nervous system

Plasticity

\section{Introduction}

Several forms of histopathological abnormalities of the enteric nervous system (ENS) and particularly of parasympathic innervation have been described. Among them, intestinal neuronal dysplasia type B (IND) and other forms of dysganglionosis such as hypoganglionosis and non-classifiable dysganglionosis (NCD) are still debated entities $[1,4,9,14,20]$.

Some authors consider IND to be a primary disease $[3,10,21]$, but it might likewise be secondary to obstruction, inflammation or other pathological conditions of the bowel $[18,19,25]$. It also has ill-defined clinical consequences. IND has been reported as an isolated lesion (i.e., not associated with other innervation disorders) $[21,26,27]$ and has been found in patients with Hirschsprung's disease (HD), proximal to the aganglionosis. It also has been blamed for the bad outcome of bowel function (BF) after the pull-through procedure (PT) $[6,22]$. In addition, hypoganglionosis has been found to be an isolated innervation disorder and to occur in association with aganglionosis in the transition zone [7, 14, 20]. It also has been mentioned as a cause of postoperative complications after surgery for HD. Milder forms of innervation changes of the ENS 
such as an increased number of ganglia and hypertrophied nerves in the submucosa (SM) have been found. The clinical significance of these forms of NCD is still puzzling as well [4].

Reports have described spontaneous favourable evolution of isolated IND [18, 26], confirmed by histopathological studies. These observations could explain reports of long-term improvement of BF in patients with a bad short-term outcome after surgery for HD [17, 24]. This clinical evolution has already been described in other reports $[2,13]$.

Our hypothesis was that HD-associated IND could evolve to normal innervation patterns. To our knowledge, this form of plasticity has not yet been confirmed by histopathological studies. We thus decided to compare the clinical follow-up of BF with the evolution of the histological patterns of the ENS in three patients who underwent surgery for HD. In order to avoid confusion and debate about IND [1], we believe that only clear criteria should be used for its diagnosis. Although other features have been described in association with IND (e.g., ectopic ganglion cells and marked acetylcholinesterase activity in the mucosa in the muscularis mucosae and around vessels [6]), our diagnosis was based on the presence of submucosal giant ganglia containing more than seven mature cells [15]. Criteria proposed by Meier-Ruge were used for the diagnosis of other types of dysganglionosis $[8,13]$.

\section{Materials and methods}

Patients

Since 1995 , we have prospectively introduced a systematic assessment of the ENS of the ganglionated bowel [16] in patients operated on for HD. Between 1995 and 2002, 31 patients were operated on for HD at our institution. In eight patients, IND was found in association with HD. Three of these patients were included in this study because of the severity of the outcome of their BF. In two of them (cases 1 and 2) a colostomy had to be performed after the PT because of a bad outcome of the BF, and the first diagnosis of IND was made at that time. Of note, colostomies had to be performed in these patients because a cautious follow-up could not be ensured in their native country. Therefore, creating a colostomy seemed to be the only safe method to avoid enterocolitis, which could have been potentially fatal. Biopsies were performed prior to PT in the last patient (case 3), who was included in a prospective study that comprised the assessment of innervation above the aganglionic zone (AZ) with serial full-thickness biopsies before proceeding to PT [16].

\section{Histopathological evaluation}

Intraoperative evaluation of the innervation was carried out either with conventional hematoxylin and eosin (HE) staining or with the rapid acetyl-cholinesterase (AChE) reaction [7]. All samples were thereafter assessed with conventional AChE histochemistry according to Karnovsky and Roots [5] and immunohistochemistry for protein gene product 9.5 (PGP 9.5), a cytoplasmic marker specific for the neural system [8] and for the antigen CD56 (CD56), a killer-cell associated antigen whose expression is comparable to that of NCAM [12].

\section{Rapid AChE staining}

Each specimen was snap-frozen in Tissue-Tek OCT compound (Sakura Finetek, Torrance, USA). Frozen sections $(6 \mu \mathrm{m}$ thick) were cut serially.

The incubation medium was $40 \mathrm{mg}$ of acetylthiocholine iodide, $52 \mathrm{ml}$ of $0.1 \mathrm{mM}$ natrium acetate buffer $(\mathrm{pH} 6.0$, adjusted with $0.1 \mathrm{M}$ acetic acid), $3.8 \mathrm{ml}$ of $0.1 \mathrm{M}$ tri-natrium citrate, $4 \mathrm{ml}$ of $30 \mathrm{mM}$ copper sulfate, $1.6 \mathrm{ml}$ of $4 \mathrm{mM}$ tetraisopropyl pyrophosphoramide (iso-OMPA), $8 \mathrm{ml}$ of distilled water and $8 \mathrm{ml}$ of $5 \mathrm{mM}$ potassium hexacyanoferrate III. The sections were incubated at $37^{\circ} \mathrm{C}$ for $10 \mathrm{~min}$ in this medium and washed in distilled water. They were then treated by freshly prepared 3,3'-Diaminobenzidine-tetrahydrochloride dihydrate (DAB, Fluka Chemie, Buchs, Switzerland), $25 \mathrm{mg}$ in $100 \mathrm{ml}$ Tris buffered saline (TBS: $0.05 \mathrm{M}$ Tris, $0.9 \%$ sodium chloride, $\mathrm{pH} 7.6$ ) containing $0.015 \%$ hydrogen peroxide for $5 \mathrm{~min}$, rinsed in tap water and counterstained in Mayer's acid-free hematoxylin, dehydrated and mounted in Eukitt.

\section{Tissue preparation for immunohistochemistry}

The tissue was fixed in buffered formalin $(4 \%$ paraformaldehyde solution in phosphate buffered saline), dehydrated and embedded in paraffin wax. Paraffin sections $(6 \mu \mathrm{m}$ thick) were cut and mounted on 3-aminopropyl-triethoxysilane-coated glass slides, deparaffinized in xylol, immersed for $45 \mathrm{~min}$ in methanol with $0.3 \%$ hydrogen peroxide to block endogenous peroxidase activity and rehydrated through graded alcohols. Sections were then subjected to microwave pre-treatment in $10 \mathrm{mM}$ citrate buffer $(\mathrm{pH} 6)$ for $20 \mathrm{~min}$, according to the method of Shi et al. [23] and rinsed in TBS.

Immunohistochemistry antibodies

For the CD56 immunodetection, the first antibody was a mouse anti-CD56 monoclonal antibody (1:50, CD56, clone 1B6; Novocastra, Newcastle upon Tyne, UK), and the second antibody was a biotinylated horse antimouse $\operatorname{IgG}$ (1:200, Vector Laboratories, Burlingame, USA).

For the PGP9.5 immunodetection the first antibody was a rabbit anti-PGP 9.5 polyclonal antibody (1:800, PGP 9.5, Anawa, Wangen, Switzerland), and the second antibody was a biotinylated goat antirabbit IgG (1:400, Vector Laboratories).

Immunohistochemical procedures

To reduce non-specific antibody binding, the sections were incubated in normal horse serum for CD56 and normal goat serum for PGP 9.5 (1:30 in TBS) for $10 \mathrm{~min}$, then with the first antibody mixed in TBS containing 5\% non-fat dried milk for $30 \mathrm{~min}$ at room temperature. They were then washed in TBS and incubated with the second antibody for $30 \mathrm{~min}$ at room temperature. After washing in TBS, sections were incubated in avidin-biotin-horseradish peroxidase complex according to the manufacturer's indications (Vectastain ABC kit, Vector Laboratories). Peroxidase was visualised by freshly prepared DAB, $50 \mathrm{mg}$ in $100 \mathrm{ml}$ TBS containing $0.01 \%$ hydrogen peroxide and counterstained in Mayer's acid-free hematoxylin, dehydrated and mounted in Eukitt. Negative controls were performed using the procedure described above omitting the first antibody.

\section{Results}

All three patients had a neo-natal onset of symptoms. IND was found in serial biopsies harvested above the AZ. In all sections, diagnosis of IND was made on the 


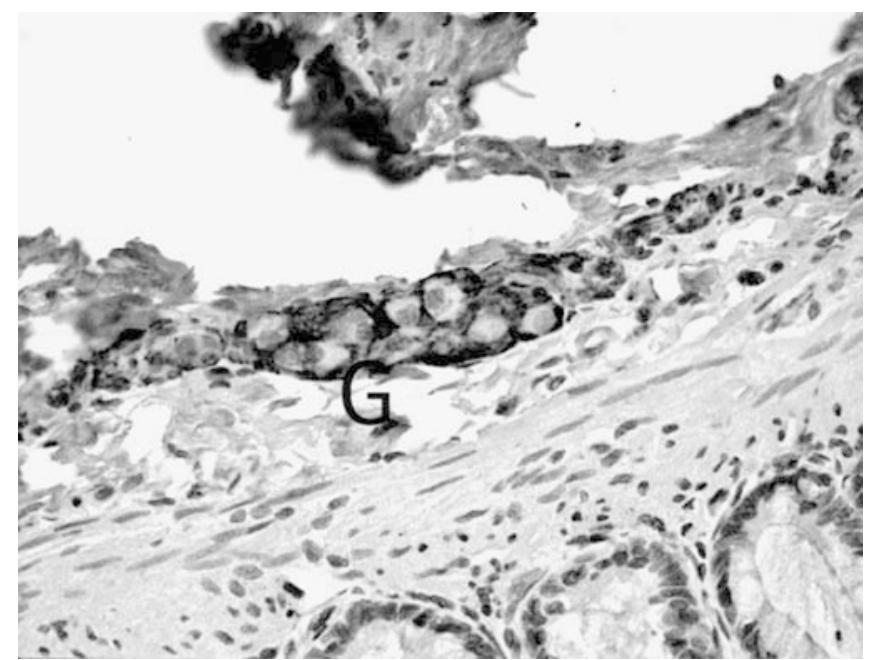

Fig. 1 Case 1. Transverse colon. Submucosal giant ganglion $(G)$ (CD 56×200)

basis of the presence of submucosal giant ganglia containing more than seven mature cells. Accompanying submucosal marked nerve hypertrophy was seen in all instances.

\section{Case 1}

The patient was referred to our institution with a colostomy that had been created on the descending colon by 2 months of age. He underwent PT 6 months later. Aganglionosis was $11 \mathrm{~cm}$ long, and intraoperative HE staining showed a normal innervation above the AZ, but postoperative $\mathrm{AChE}$ and immunohistochemistry made on the resected colon displayed IND up to $10 \mathrm{~cm}$ above the AZ (Figs. 1, 2A). Postoperatively, the patient had severe constipation and multiple episodes of enterocolitis that mandated a colostomy 1 month later. During this procedure, five full-thickness colon biopsies

Fig. 2 Case 1. Histopathology of biopsies: (A) at time of pullthough $(P T),($ B) 1 month after PT, (C) 7 months after PT and (D) 18 months after PT. Measurements taken above the excavatio rectovesicalis. ER Excavatio rectovesicalis, $A Z$ aganglionic zone, $I N D$ intestinal neuronal dysplasia, $M N H$ marked nerve hypertrophy, NH moderate nerve hypertrophy. Arrow indicates level of resection, oval represents colostomy

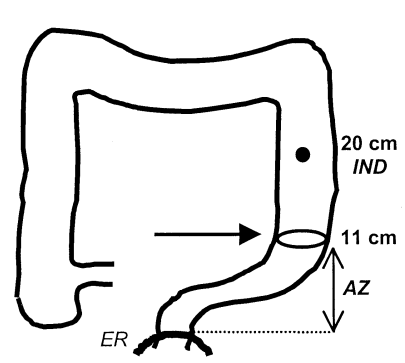

A

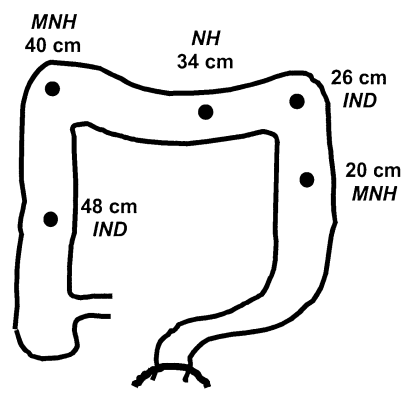

$\mathrm{B}$

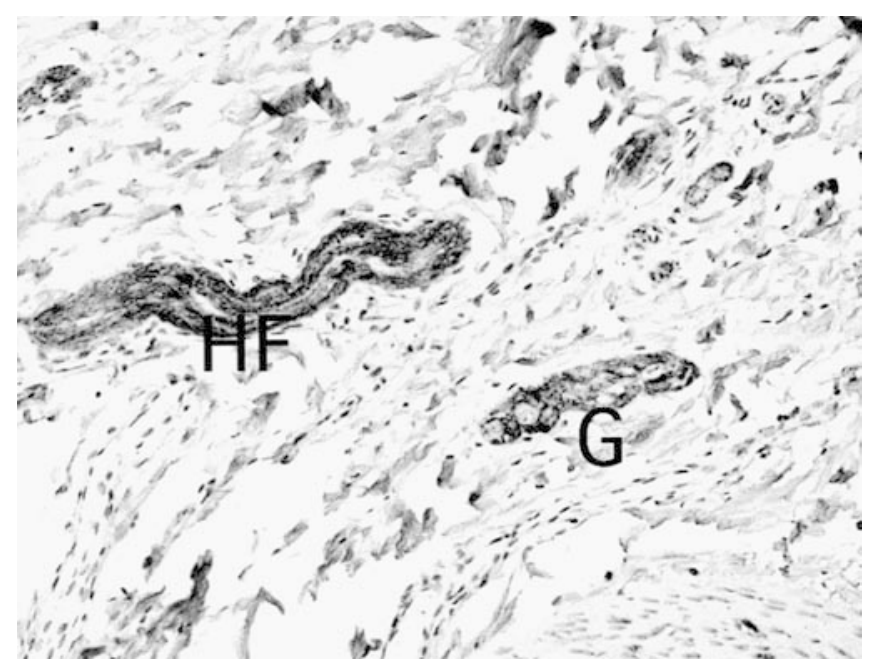

Fig. 3 Case 1. Transverse colon. Submucosal ganglion $(G)$ and markedly hypertrophied nerve fiber $(H F)$ (PGP 9.5×100)

$5 \times 10 \mathrm{~mm}$ each were harvested up to the caecum. They displayed mosaic-like innervation changes with IND in two biopsies (Fig. 2B) and marked nerve hypertrophy in the SM in three. Six months later, biopsies were repeated at the same levels as before. At that time, a marked nerve hypertrophy was seen in the SM without criteria for IND (Figs. 2C, 3). The colostomy was then closed, but once again the patient had severe constipation and multiple bouts of enterocolitis. It was decided to place a new colostomy and to keep it for a longer period of time. Biopsies were repeated after 18 months. At that time, only a moderate nerve hypertrophy was seen and the colostomy was closed (Figs. 2D, 4). The patient had normal bowel movements and good continence with follow-up of 4 years.

\section{Case 2}

The patient was referred to our institution with a colostomy that had been created by 10 months of age on the mid-descending colon. PT was performed 4 months later. The colon was massively dilated above the colostomy. The AZ was $35 \mathrm{~cm}$ long, and intraoperative biopsies assessed with rapid AChE staining showed that colostomy had been made in the AZ. Serial full-thickness biopsies also displayed IND 10 and $24 \mathrm{~cm}$ above
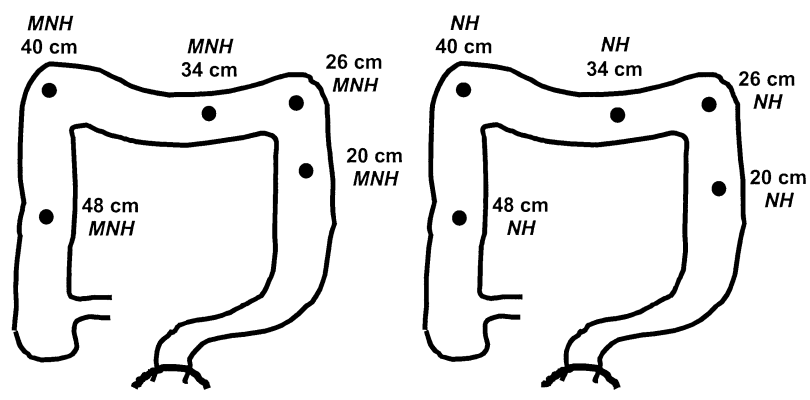

C 


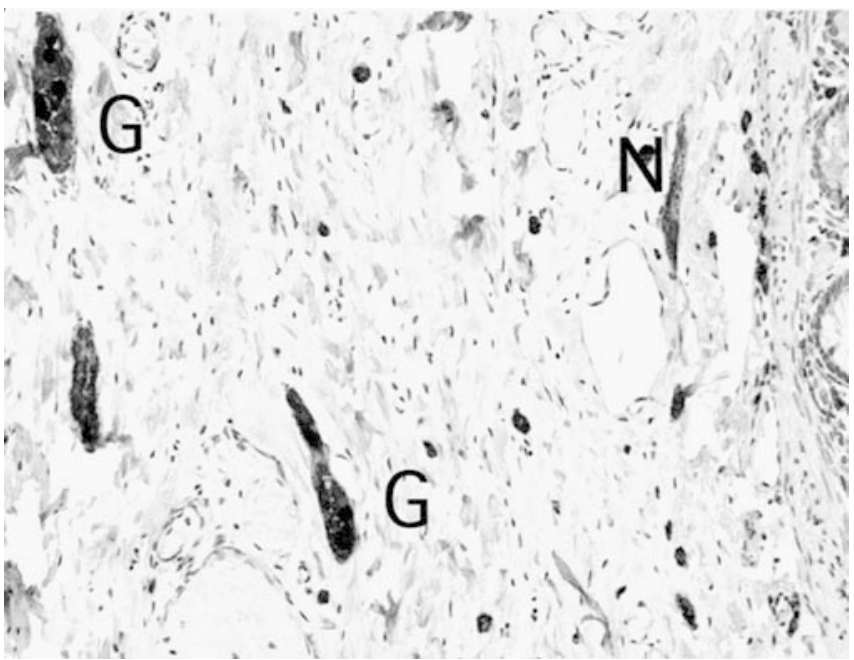

Fig. 4 Case 1. Transverse colon. Normal submucosal ganglions $(G)$ and moderately hypertrophied nerve fiber $(N)$ (PGP 9.5×100)

the AZ (Fig. 5A). Because of multiple episodes of enterocolitis and severe constipation in spite of conservative treatment for 1 month, we decided in favour of a colostomy. During this procedure, three biopsies were harvested along the colon. They all confirmed the presence of IND (Fig. 5B). The patient kept the colostomy for 1 year. At this time, renewed biopsies were made at the same levels as before. They all showed a normal innervation (Fig. 5C). The colostomy was consequently closed. With a follow-up of 4 years, the patient had good continence and daily bowel movements.

Case 3

This patient was referred to our institution as a neonate. After confirmation of HD with rectal suction biopsies, she was first managed with rectal washouts. By 2.5 months of age, we first assessed the colon innervation with five full-thickness colon biopsies that showed a 15-

Fig. 5 Case 2. Histopathology of biopsies: (A) at time of pullthrough $(P T)$, (B) 1 month after PT and (C) 1 year after PT. Measurements taken above the excavatio rectovesicalis. ER Excavatio rectovesicalis, $A Z$ aganglionic zone, IND intestinal neuronal dysplasia. Arrow indicates level of resection, oval represents colostomy cm-long aganglionosis and IND 10 and $15 \mathrm{~cm}$ above the AZ (Fig. 6A). A colostomy was then created. After 9 months, serial biopsies were repeated at the same levels as before. They all showed a normal innervation with only moderate nerve hypertrophy in two. PT was thus undertaken. Immunohistopathological examination of the resected colon displayed the presence of IND limited to $3 \mathrm{~cm}$ above the $\mathrm{AZ}$ and to the proximal stoma of the colostomy (Fig. 6B). The patient has normal BF with 6 years of follow-up.

\section{Discussion}

Dysganglionosis consists of a broad spectrum of innervation disorders that may be associated in the same patient. On one side of the spectrum, hypoganglionosis might represent the transition between normal innervation and aganglionosis. When ignored, it can be the cause of complications after surgery for $\operatorname{HD}[7,14,20]$.

On the other side of the spectrum, IND type B is characterised by a hyperganglionosis and hypertrophied nerves in the SM, as well as ectopic nerve cells in the LP $[8,13,15]$. It has been described as an isolated disorder $[10,21,26,27]$ and reported in association with other pathological conditions of the bowel like necrotising enterocolitis, mucoviscidosis, bowel obstruction in neonates and anorectal malformations with secondary megacolon or constipation [18, 19, 25]. IND could therefore be considered either as a cause or a conse-

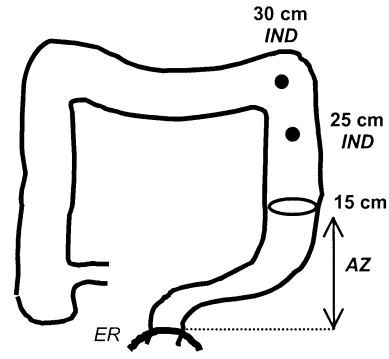

A

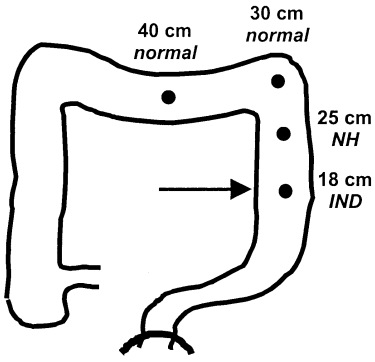

B
Fig. 6 Case 3. Histopathology of biopsies: (A) at time of biopsies and colostomy and (B) after 9 months at the time of pull-through. Measurements taken above the excavatio rectovaginalis. $E R$ Excavatio rectovaginalis, $A Z$ aganglionic zone, IND intestinal neuronal dysplasia, $N H$ moderate nerve hypertrophy. Oval represents colostomy, arrow indicates level of resection

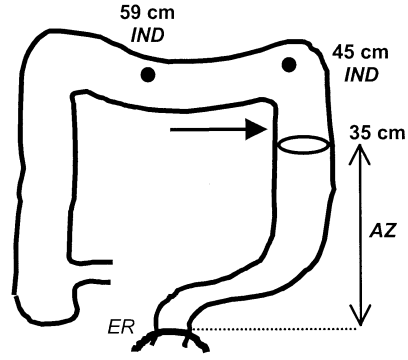

A

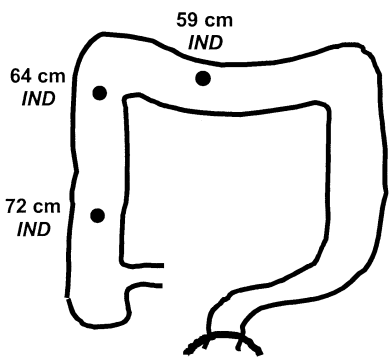

B

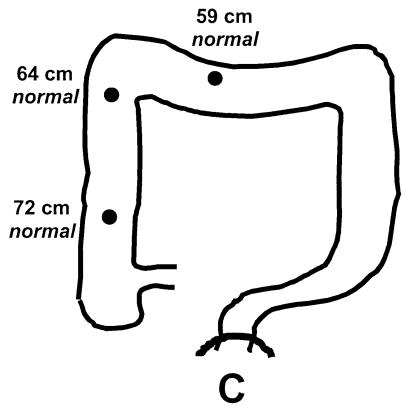


quence of these diseases. Also, it has been encountered in as many as $40 \%$ of patients with $\mathrm{HD}$, proximal to the $\mathrm{AZ}$ and blamed for the bad postoperative outcome of $\mathrm{BF}$ in these patients $[6,22]$.

These innervation changes remain controversial $[1,9]$. This in part could be due to the sophisticated methods required to assess them, such as the acetyl-cholinesterase histochemistry and immunohistochemistry techniques, but also to the lack of agreement in the criteria used to classify these changes. Of note, our experience corroborates the reports of overlooked dysganglionosis when no more than "conventional" staining techniques like HE were used [6,7]. Rapid AChE permits an intraoperative exploration of the innervation, but the final diagnosis should be confirmed with specific histopathological techniques like conventional AChE after Karnovsky and Roots and immunohistochemistry for PGP 9.5 and CD56. We would therefore suggest applying these diagnostic methods on fullthickness biopsies as soon as innervation disorders are suspected.

Simpser et al. [26] have reported the spontaneous regression of isolated IND in one patient. Same reports were made by Rintala and al. in infants with bowel obstruction [18]. Since 1995, we prospectively assessed the ENS of the ganglionated bowel above the AZ at the time of PT. From 1995 to 2002, 31 patients were operated on for HD in our institution. Nine had severe constipation after PT, and IND was found in eight of them. In all of these patients, BF improved in a period of time ranging from 1 to 5 years. In three patients who were repeatedly histologically reassessed because of the severity of their complications, this particular dysganglionosis evolved to normal innervation within a period ranging from 9 to 18 months. In one patient (case 1), this evolution occurred stepwise. In the biopsies repeated after 6 months, criteria for IND were not met, but changes of the innervation such as a marked nerve hypertrophy in the SM were still present. Considering that they were not found in the biopsies repeated after 18 months, these changes may represent a milder form of hyperplasia of the parasympathic nervous system and be intermediate innervation patterns between IND and normal innervation. The moderate nerve hypertrophy that was encountered in two patients did not have any clinical implication when seen as an isolated finding. Nevertheless, when found in a patient with disorders of the $\mathrm{BF}$, it should raise the suspicion of the presence of more severe types of dysganglionosis coexisting in the same region of the bowel, and this should be searched for.

These observations have led us to the hypothesis that IND could be responsible for the bad postoperative short-term outcome of $\mathrm{BF}$ in some patients with $\mathrm{HD}$ and that the long-term improvement of BF reported by some authors [17, 24] might be explained by the normalisation of innervation patterns of the colon proximal to the aganglionosis, at least in some of these patients.
Distension or inflammation of the bowel wall, as well as stool retention, can explain the development or the persistence of IND. Colostomies that had been placed to avoid stool retention and consequent dilatation of the bowel may therefore have contributed to the plasticity encountered in our patients. Marty et al. [11] have successfully proposed postoperative repeated irrigations in all patients operated on for HD in order to reduce postoperative complications. This method may have had the same result as a colostomy on the bowel wall and thus on ENS.

Although some authors have considered IND as a primary disease and proposed resection of the involved bowel segments [2, 27], others have suggested conservative treatment with saline washouts $[21,26]$. Our study shows that IND might evolve to normal innervation. Also, postnatal changes in the neuronal density of the myenteric plexus have been described [28], and IND changes might be part of this overall evolution. Dealing with this innervation disorder therefore requires being aware of its ability to have a certain degree of plasticity in order to avoid preventable major surgical procedures. The finding of IND is an indication for daily bowel washouts, or for a colostomy in selected cases. According to the outcome of BF, biopsies should thereafter be repeated after a delay of about 12 months in order to assess the evolution of innervation patterns.

\section{References}

1. Csury L, Peña A (1995) Intestinal neuronal dysplasia. Myth or reality? Literature review. Pediatr Surg Int 10:441-446

2. Fadda B, Pistor G, Meier-Ruge W, Hofmann-von Kap-herr S, Müntefering H, Espinoza R (1987) Symptoms, daignosis, and therapy of neuronal intestinal dysplasia masked by Hirschsprung's disease. Pediatr Surg Int 2:76-80

3. Giaroni C, De Ponti F, Cosentino M, Lecchini S, Frigo G (1999) Plasticity of the enteric nervous system. Gastroenterology 117:1438-1458

4. Hohlschneider AM, Meier-Ruge W, Ure BM (1994) Hirschsprung's disease and allied disorders- A review. Eur J Pediatr Surg 4:260-266

5. Karnovsky MJ, Roots L (1965) A "direct-coloring" thiocholine method for cholinesterases. J Histochem Cytochem 12:219-221

6. Kobayashi H, Hirakawa H, Surana R, O'Briain DS, Puri P (1995) Intestinal neuronal dysplasia is a possible cause of persistent bowel symptoms after pull-through operation for Hirschsprung's disease. J Pediatr Surg 30:253-259

7. Kobayashi $\mathrm{H}$, Wang $\mathrm{Y}$, Hirakawa $\mathrm{H}$, O'Briain DS, Puri $\mathrm{P}$ (1995) Intraoperative evaluation of extent of aganglionosis by a rapid acetylcholinesterase histochemical technique. J Pediatr Surg 30:248-252

8. Krammer HJ, Meier-Ruge W, Sigge W, Eggers R, Kühnel W (1994) Histopathological features of neuronal intestinal dysplasia of the plexus submucosus in whole mounts revealed by immunohistochemistry for PGP 9.5. Eur J Pediatr Surg 4:358-361

9. Lake BD (1995) Intestinal neuronal dysplasia. Why does it only occur in parts of Europe ? Virchows Arch 426:537-539

10. Martuciello G, Caffarena PE, Lerone M, Mattioli G, Barabino A, Bisio G, Jasonni V (1994) Neuronal intestinal dysplasia: Clinical experience in italian patients. Eur J Pediatr Surg 4:287292 
11. Marty TL, Seo T, Sullivan JJ, Matlak ME, Black RE, Johnson DG (1995) Rectal irrigations for the prevention of postoperative enterocolitis in Hirschsprung's disease. J Pediatr Surg 30:652-654

12. Mechtersheimer G, Staudter M, Möller P (1991) Expression of the natural killer-cell associated antigens CD56 and CD57 in human neural and striated muscle cells and their tumors. Cancer Res 51:1300-1307

13. Meier-Ruge W, Gambazzi F, Kaüfeler RE, Schmid P, Schmidt CP (1994) The neuropathological diagnosis of neuronal intestinal dysplasia (NID B). Eur J Pediatr Surg 4:267-273

14. Meier-Ruge WA, Brunner LA, Engert J, Heminghaus M, Hohlschneider AM, Jordan P, Piket G, Posselt HG, Schärli A (1999) A correlative morphometric and clinical investigation of hypoganglionosis of the colon in children. Eur J Pediatr Surg 9:67-74

15. Meier-Ruge WA, Longo-Bauer CH (1997) Morphometric determination of the methodological criteria for the diagnosis of intestinal neuronal dysplasia (IND B). Pathol Res Pract 193:465-469

16. Meyrat BJ, Lesbros Y, Laurini RN (2001) Assessment of the colon innervation with serial biopsies above the aganglionic zone before the pull-through procedure in Hirschsprung's disease. Pediatr Surg Int 17:129-135

17. Moore SW, Millar AJW, Cywes S (1994) Long-terme clinical, manometric, and histological evaluation of obstructive symptoms in the postoperative Hirschsprung's patient. J Pediatr Surg 29:106-111

18. Rintala R, Rapola J, Louhimo I (1989) Neuronal intestinal dysplasia. Progr Pediatr Surg 24:186-192

19. Sacher P, Briner J, Hanimann B (1993) Is intestinal neuronal dysplasia (IND) a primary disease or a secondary phenomenon ? Eur J Pediatr Surg 3:228-230
20. Schärli AF, Sossai R (1998) Hypoganglionosis. Sem Pediatr Surg 7:187-191

21. Schmittenbecher PP, Sacher P, Cholewa D, Haberlik A, Menardi G, Moczulski J, Rumlova E, Schuppert W, Ure B (1999) Hirschsprung's disease and intestinal neuronal dysplasia: A frequent association with implications for the postoperative course. Pediatr Surg Int 15:553-558

22. Schmittenbecher PP, Gluck M, Wiebecke B, Meier-Ruge W (2000) Clinical long-term results in intestinal neuronal dysplasia (IND). Eur J pediatr Surg 10:17-22

23. Shi SR, Key ME, Karla KL (1991) Antigen retrieval in formalin-fixed, paraffin-embedded tissues: An enhancement method for immunohistochemical staining based on microwave oven heating on tissue sections. J Histochem Cytochem 39:741748

24. Shono K, Hutson JM (1994) The treatment and postoperative complications of Hirschsprung's disease. Pediatr Surg Int 9:362-365

25. Sigge W, Wedel T, Kuhnel W, Krammer HJ (1998) Morphologic alterations of the enteric nervous system and deficiency of non-adrenergic non-cholinergic inhibitory innervation in neonatal necrotizing enterocolitis. Eur $\mathbf{J}$ pediatr Surg 8:87-94

26. Simpser E, Kahn E, Kenigsberg K, Duffy L, Markowitz J, Daum F (1991) Neuronal Intestinal Dysplasia: Quantitative diagnostic criteria and clinical management. J Pediatr Gastroenterol Nutr 12:61-64

27. Ure BM, Holschneider AM, Meier-Ruge W (1993) Neuronal intestinal malformations: A retro- and prospective study on 203 patients. Eur J Pediatr Surg 4:279-286

28. Wester T, O’Brian DS, Puri P (1999) Notable postnatal alterations in the myenteric plexus of normal human bowel. Gut 44:666-674 